\title{
Epidemiological and Treatment Trends for Acute Ischemic Stroke Preceding and during the COVID-19 Pandemic
}

\author{
Adrian Rodrigues Michael C. Jin Arjun Pendharkar \\ Department of Neurosurgery, Stanford School of Medicine, Stanford, CA, USA
}

\section{Keywords}

Stroke - Acute ischemic stroke - Mechanical thrombectomy . Coronavirus disease 2019. Socioeconomic

\begin{abstract}
Background: The coronavirus disease 2019 (COVID-19) pandemic heralded a number of indirect perturbations to patient behavior and disease epidemiology, and mounting evidence suggests that the COVID-19 pandemic may have exacerbated underlying health disparities along racial and socioeconomic (SES) groups for acute ischemic stroke (AIS). We used 1 large national insurance database to identify whether patient demographics, disease severity, or mechanical thrombectomy (MT) rates changed for the treatment and management of AIS during COVID-19. Methods: AIS patient records were queried from the Clinformatics ${ }^{\circledR}$ Data Mart Optum SES Database from the following 2 time periods: March 1, 2019-June 30, 2019 (pre-COVID-19), and March 1, 2020-June 30, 2020 (COVID-19). The database contains the longitudinal healthcare claims of approximately 77 million patients covered by a major insurance provider between 2003 and June 30, 2020 across all 50 states. Interrupted timeseries analyses were used to assess trend differences before and after the COVID-19 pandemic. Results: During the preCOVID-19 period (March 1, 2019-June 30, 2019), there were 9,072 patients who presented for AIS, compared to 7,366 during COVID-19 (March 1, 2020-June 30, 2020). In both
\end{abstract}

karger@karger.com www.karger.com/ced

Karger"

GOPEN ACCESS
(C) 2021 The Author(s).

Published by S. Karger AG, Basel

This is an Open Access article licensed under the Creative Commons Attribution-NonCommercial-4.0 International License (CC BY-NC) (http://www.karger.com/Services/OpenAccessLicense), applicable to the online version of the article only. Usage and distribution for commercial purposes requires written permission. periods, the majority of patients were white $(66.83 \%$ preCOVID-19 and $67.91 \%$ during COVID-19). The average hospitalization duration was not different during the 2 time periods $(p=0.632)$, nor were rates of MT $(p=0.260)$. Total inpatient costs rose slightly for the COVID-19 period (USD 30,739 vs. USD 29,406; $p=0.015$ ), and the median National Institutes of Health Stroke Scale (NIHSS) score was higher during COVID-19 ( 5 vs. $4 ; p=0.023$ ). When longitudinal trends were assessed for rates of MT and average NIHSS score for black and white patients, no differences were noted during the COVID-19 pandemic. Patients without any undergraduate experience did not present with AIS in increasing or decreasing incidence during COVID-19 $(p=0.268)$, but they did undergo declining rates of MT ( $p=0.013)$. Conclusions: In the largest SES analysis of AIS patients during the COVID-19 era, we found that several SES factors, including race and income, did not seem to significantly impact utilization of MT for the treatment of AIS or the severity of the stroke at presentation.

(c) 2021 The Author(s)

Published by S. Karger AG, Basel

\section{Introduction}

The coronavirus disease 2019 (COVID-19) pandemic heralded a number of indirect perturbations on patient behavior and disease epidemiology, and mounting evidence suggests that the COVID-19 pandemic exacerbated 
underlying health disparities along racial and socioeconomic (SES) groups [1]. In particular, the treatment and epidemiology of acute ischemic stroke (AIS) during COVID-19 has been an area of dedicated study [2-4]. While the delay-to-care and reduction in hospital volumes for AIS is now well documented [4], more recent studies have begun to elucidate racial disparities in AIS presentation and treatment $[5,6]$. However, available approaches have been limited to single- or multi-institution studies, and no national database has been used. Well-maintained databases require time to collate and audit the data, and therefore reliable databases with information from the 2020 year have only recently become available for analysis. We used 1 large national insurance database to identify whether patient demographics, disease severity, or mechanical thrombectomy (MT) rates changed for the treatment and management of AIS during COVID-19.

\section{Methods}

Patient records were queried from the Clinformatics ${ }^{\circledR}$ Data Mart Optum SES Database from the following 2 time periods: March 1, 2019-June 30, 2019 (pre-COVID-19) and March 1, 2020-June 30, 2020 (COVID-19). The database contains the longitudinal healthcare claims of approximately 77 million patients covered by a major insurance provider between 2003 and June 30, 2020 across all 50 states. All records are verified, adjudicated, adjusted, and de-identified prior to inclusion into the database. Data are updated semi-annually, and all data are de-identified. This study was approved by our Institutional Review Board (\#40974).

Patients diagnosed with AIS were identified via the ICD-10 code I63.X. Patients with $<2$ day hospitalization were excluded (online suppl. Fig. 1; for all online suppl. material, see www. karger.com/doi/10.1159/000518935). Patients who were transferred between $2+$ hospitals were counted as 1 hospitalization. The National Institutes of Health Stroke Scale (NIHSS) score was extracted with the ICD-10 code R27.X.

Interrupted time series analyses were used to assess trend differences before and after the COVID-19 pandemic. $\chi^{2}$ and Student's $t$ tests were used to compare categorical and continuous variables as appropriate. Statistical analyses were conducted in $\mathrm{R}$ Studio version 1.0.153 and Stata/SE 16.1 (StataCorp, College Station, TX, USA). Graphical representations were created using GraphPad Prism 9.1.0.

\section{Results}

During the pre-COVID-19 period (March 1, 2019-June $30,2019)$, there were 9,072 patients who presented for AIS, compared to 7,366 during COVID-19 (March 1, 2020June 30, 2020). Differences in race, education, or income were not noted between the 2 time periods. The average hospitalization duration was not different $(p=0.632)$, nor were rates $\operatorname{MT}(p=0.260)$ (online suppl. Table 1). Total inpatient costs rose slightly for the COVID-19 period (USD $30,739$ vs. USD 29,$406 ; p=0.015)$, and the median NIHSS score was higher during COVID-19 (5 vs. 4 ; $p=0.023$ ).

When longitudinal trends were assessed for rates of MT and average NIHSS score for black and white patients, no differences were noted during the COVID-19 pandemic (Fig. 1a-d). Patients without any undergraduate experience did not present with AIS in increasing or decreasing incidence during COVID-19, but they did undergo declining rates of MT (Fig. 1e, f). Other trends stratified by patient income or category of medical service received were not significantly different during COVID-19 (online suppl. Fig. 2a, b, 3).

\section{Conclusions}

While other authors have noted treatment and epidemiological differences in AIS during COVID-19, we found few such changes in 1 large national database. We did find that patients without undergraduate experience received $\mathrm{MT}$ at reduced rates compared to patients with collegiate experience during COVID-19. However, other SES factors, including race and income, did not seem to significantly impact utilization of MT for the treatment of AIS or the severity of the stroke at presentation.

Therefore, though we were able to extract over 16,000 AIS patient records and assess static and longitudinal differences during the COVID-19 era, we found very few noteworthy changes. Though costs and the average NIHSS score rose slightly, the increases are not likely to be clinically significant. Since the findings of this study run counter to the existing literature, it is important to enumerate possible explanations. First, previous studies, given the limited available data, relied upon single or multi-institution patient registries and were not able to obtain nationally representative data. Second, our inclusion criteria do not mirror those of previous efforts. Our study included only AIS patients and those who were admitted 2+ days. We did not look at hemorrhagic stroke or patients who were not hospitalized overnight. Third, it is possible that racial and sociodemographic trends are less substantial when only insured patients are considered, rather than the general public or those treated at academic centers, for example. Indeed, it was reassuring that racial or income differences were not noted in the weekly incidence of AIS or use of MT. While we did find that fewer AIS patients presented during the COVID-19 era, 


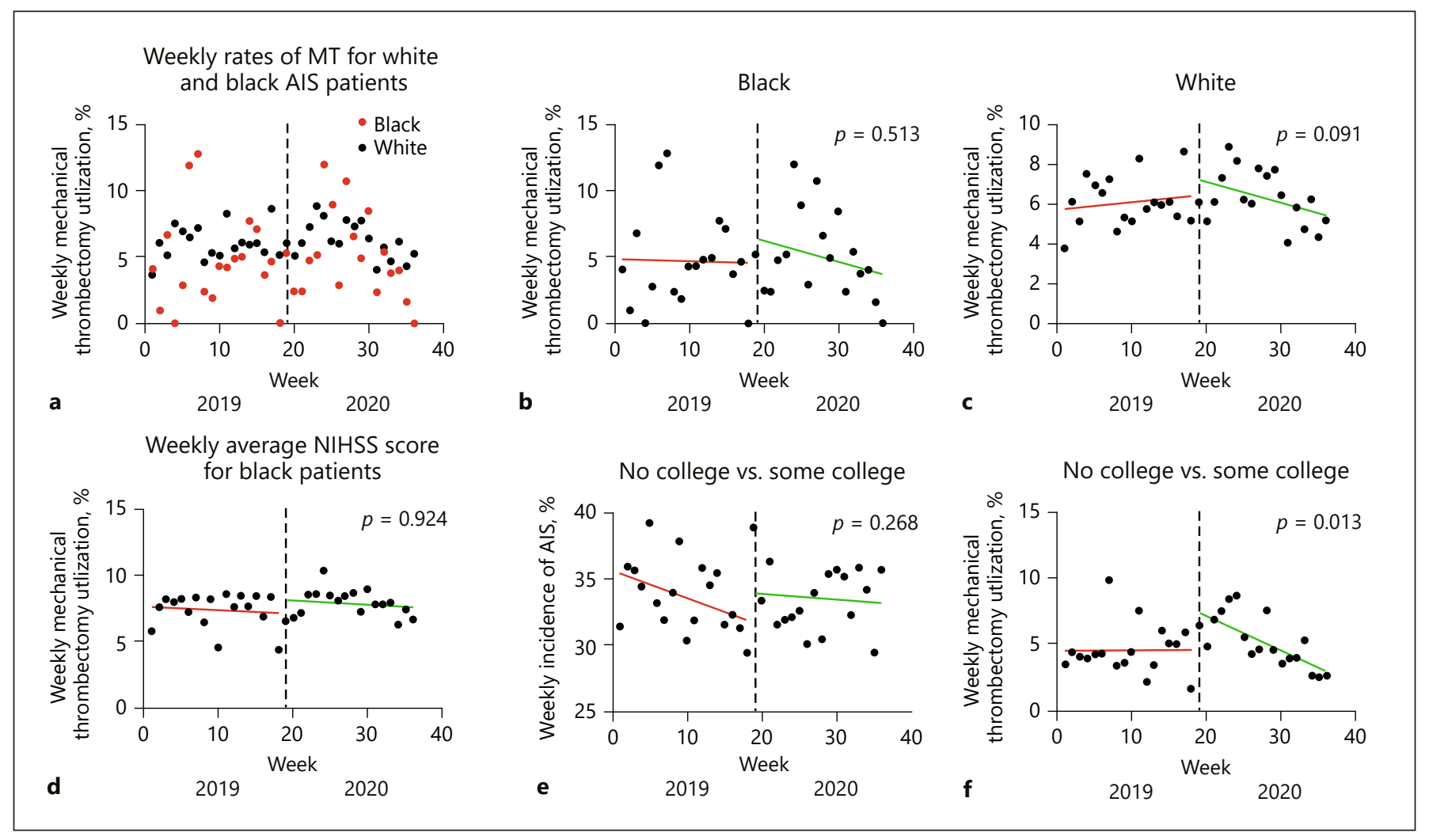

Fig. 1. a The percentage of AIS patients each week who underwent MT, stratified by black or white race. The year 2019 is separated into weeks 1-18 (March 01, 2019-June 30, 2019) and the year 2020 is separated into weeks 19-36 (March 01, 2020-June $30,2020)$. b, c The percentage of black and white AIS patients each week who underwent MT with interrupted time series trendlines interpolated in each treatment era (March 01, 2019June 30, 2019 vs. March 01, 2020-June 30, 2020). d The average NIHSS score for black AIS patients each week with interrupted

as others have noted [7], the patient population who did present to the hospital were typical of pre-COVID-19 epidemiology. Thus, while hospital AIS volumes dropped during COVID-19, the patient groups who did arrive at a medical facility were consistent with the pre-COVID-19 racial and sociodemographic trends.

Our study's findings should be tempered by the limitations inherent to retrospective database analyses. First, selection bias is a concern. Our insurance claims database, while large, may not be representative of all patient populations (e.g., uninsured patients). As more data become available from the COVID-19 era, further retrospective study is warranted. Second, we rely upon the accurate coding of diagnoses, procedures, and SES variables. Though data within the Optum SES registry are closely reviewed, adjudicated, and verified, it is possible

Trends for Acute Ischemic Stroke during the COVID-19 Pandemic time series trendlines interpolated in each treatment era. e The percentage of AIS patients without any undergraduate experience each week with interrupted time series trendlines interpolated in each treatment era. $\mathbf{f}$ The percentage of AIS patients without any undergraduate experience score each week who underwent MT with interrupted time series trendlines interpolated in each treatment era. MT, mechanical thrombectomy; AIS, acute ischemic stroke; NIHSS, National Institutes of Health Stroke Scale.

that errors remain. Given our large sample size, however, the effect of individual errors is unlikely to be large. Third, we capture only data from the beginning of the COVID-19 pandemic, as more recent data are unavailable. It is possible that the SES trends observed in the present study have changed after June 30, 2020. Without more complete data, this is impossible to state with certainty, and we do present the largest cohort of AIS patients during the COVID-19 pandemic thus far.

In summary, we were able to extract over 16,000 AIS patient records and assess static and longitudinal differences in SES patient trends during the COVID-19 era. We found very few noteworthy changes. Though costs and the average NIHSS score rose slightly, the increases are not likely to be clinically significant. Therefore, in the largest SES analysis of AIS patients during the COVID-19 era, we found that 
several SES factors, including race and income, did not seem to significantly impact utilization of MT for the treatment of AIS or the severity of the stroke at presentation.

\section{Acknowledgments}

Data for this project were accessed using the Stanford Center for Population Health Sciences Data Core. The PHS Data Core is supported by a National Institutes of Health National Center for Advancing Translational Science Clinical and Translational Science Award (UL1 TR001085) and from Internal Stanford funding. The content is solely the responsibility of the authors and does not necessarily represent the official views of the NIH.

\section{Statement of Ethics}

All records are verified, adjudicated, adjusted, and de-identified prior to inclusion into the database. Data are updated semiannually, and all data are de-identified. This study was approved by our Institutional Review Board (\#40974).

\section{Conflict of Interest Statement}

The authors have no conflicts of interest.

\section{Funding Sources}

The authors have no funding sources, financial support, or industry affiliation to disclose.

\section{Author Contributions}

A.R. and M.C.J. involved in study design, data acquisition, and data analysis; A.R. involved in manuscript writing; and A.P. involved in study supervision and revision.

\section{Data Availability Statement}

Accessed through the Optum SES database. All ICD and CPT codes are publicly available.

\section{References}

1 Webb Hooper M, Nápoles AM, Pérez-Stable EJ. COVID-19 and racial/ethnic disparities. JAMA. 2020;323(24):2466-7.

2 Friedlich D, Newman T, Bricker S. Retraction notice to "impact of the COVID-19 pandemic on stroke epidemiology and clinical stroke practice in the US" [Journal of Stroke and Cerebrovascular Diseases, Vol. 30, No. 4 (April), 2021: 105639]. J Stroke Cerebrovasc Dis. 2021;30(8):105982.

3 Rameez F, McCarthy P, Cheng Y, Packard LM, Davis AT, Wees N, et al. Impact of a stay-at-home order on stroke admission, subtype, and metrics during the COVID-19 pandemic. Cerebrovasc Dis Extra. 2020;10(3):159-65.

4 Logroscino G, Beghi E. Stroke epidemiology and COVID-19 pandemic. Curr Opin Neurol. 2021;34(1):3-10.

5 Al Kasab S, Almallouhi E, Alawieh A, Jabbour P, Sweid A, Starke RM, et al. Alarming downtrend in mechanical thrombectomy rates in African American patients during the COVID-19 pandemic-Insights from STAR. J Neurointerv Surg. 2021;13(4):304-7.
6 Cummings C, Almallouhi E, Al Kasab S, Spiotta AM, Holmstedt CA. Blacks are less likely to present with strokes during the COVID-19 pandemic: observations from the buckle of the stroke belt. Stroke. 2020; 51(10):3107-11

7 Bhambhvani HP, Rodrigues AJ, Yu JS, Carr JB 2nd, Hayden Gephart M. Hospital volumes of 5 medical emergencies in the COVID-19 pandemic in 2 US medical centers. JAMA Intern Med. 2020 Feb 1;181(2): 272-4. 